\title{
Transgenic and knockout analyses of Masculinizer and doublesex illuminated the unique functions of doublesex in germ cell sexual development of the silkworm, Bombyx mori
}

Tomohisa Yuzawa ${ }^{1,2}$, Misato Matsuoka ${ }^{2,3}$, Megumi Sumitani ${ }^{4}$, Fugaku Aoki ${ }^{2}$, Hideki Sezutsu ${ }^{4}$ and Masataka G. Suzuki $i^{*}$

\begin{abstract}
Background: Masculinizer (Masc) plays a pivotal role in male sex determination in the silkworm, Bombyx mori. Masc is required for male-specific splicing of $B$. mori doublesex ( $B m d s x$ ) transcripts. The male isoform of $B m d s x$ (BmdsxM) induces male differentiation in somatic cells, while females express the female isoform of Bmdsx (BmdsxF), which promotes female differentiation in somatic cells. Our previous findings suggest that Masc could direct the differentiation of genetically female (ZW) germ cells into sperms. However, it remains unclear whether Masc directly induces spermatogenesis or if it promotes male differentiation in germ cells indirectly by inducing the expression of BmdsxM.

Results: In this study, we performed genetic analyses using the transgenic line that expressed Masc, as well as various Bmdsx knockout lines. We found that Masc-expressing females with a homozygous mutation in BmdsxM showed normal development in ovaries. The formation of testis-like tissues was abolished in these females. On the other hand, Mascexpressing females carrying a homozygous mutation in BmdsxF exhibited almost complete male-specific development in gonads and germ cells. These results suggest that BmdsxM has an ability to induce male development in germ cells as well as internal genital organs, while BmdsxF inhibits BmdsxM activity and represses male differentiation. To investigate whether MASC directly controls male-specific splicing of Bmdsx and identify RNAs that form complexes with MASC in testes, we performed RNA immunoprecipitation (RIP) using an anti-MASC antibody. We found that MASC formed a complex with AS1 IncRNA, which is a testis-specific factor involved in the male-specific splicing of Bmdsx pre-mRNA. (Continued on next page)
\end{abstract}

\footnotetext{
* Correspondence: gakyo@edu.k.u-tokyo.ac.jp

2Department of Integrated Biosciences, Graduate School of Frontier Sciences,

The University of Tokyo, 5-1-5 Kashiwanoha, Kashiwa-shi, Chiba 277-8562,

Japan

Full list of author information is available at the end of the article
}

(C) The Author(s). 2020 Open Access This article is licensed under a Creative Commons Attribution 4.0 International License, which permits use, sharing, adaptation, distribution and reproduction in any medium or format, as long as you give appropriate credit to the original author(s) and the source, provide a link to the Creative Commons licence, and indicate if changes were made. The images or other third party material in this article are included in the article's Creative Commons licence, unless indicated otherwise in a credit line to the material. If material is not included in the article's Creative Commons licence and your intended use is not permitted by statutory regulation or exceeds the permitted use, you will need to obtain permission directly from the copyright holder. To view a copy of this licence, visit http://creativecommons.org/licenses/by/4.0/ The Creative Commons Public Domain Dedication waiver (http://creativecommons.org/publicdomain/zero/1.0/) applies to the data made available in this article, unless otherwise stated in a credit line to the data. 
(Continued from previous page)

Conclusions: Taken together, our findings suggest that Masc induces male differentiation in germ cells by enhancing the production of BmdsxM. Physical interaction between MASC and AS1 IncRNA may be important for the BmdsxM expression in the testis. Unlike in the Drosophila dsx, BmdsxM was able to induce spermatogenesis in genetically female $(Z W)$ germ cells. To the best of our knowledge, this is the first report that the role of $d s x$ in germ cell sexual development is different between insect species.

Keywords: Bombyx mori, Sex determination, Sexual differentiation, Masc, Bmdsx, Gonad, Germ cell, Alternative splicing

\section{Background}

Sex determination and sexual differentiation are strictly controlled by the sex-determination cascade that is composed of various proteins encoded by sex-determining genes. Although in all animal species, the females produce eggs as gametes while the males produce spermatozoa, there is high diversity in sex-determining genes among animal species [1]. For example, in vertebrates, the Sex-determining region Y (SRY) functions as a determinant of male differentiation in mammals [2]. In rainbow trout, the sex-determining region $Y(S d Y)$ [3], which is homologous to the immune system gene interferon regulatory factor 9 (IRF9), triggers masculinization. In insects, the upstream regulators for maleness also exhibit significant diversity among species. A male determining factor Nix (Nix) in the mosquito Aedes aegypti [4], a maleness gene $Y o b$ in the malaria mosquito Anopheles gambiae [5], and the Musca domestica male determiner $(M d m d)$ in the housefly [6] differ in origin, structure, and mechanism of action.

In the silkworm, the chromosomal sex determination mechanism is distinct from those of mammals, mosquitoes and flies, with the female (ZW) being the heterogametic sex and the male (ZZ) the homogametic sex [7]. Accumulating evidence indicates that a Z-linked gene Masculinizer (Masc) acts as a key determinant for maleness in the silkworm $[8,9]$. In individuals without the W chromosome, Masc induces male-specific splicing of the pre-mRNA of the Insulin-like growth factor II mRNA binding protein (Imp) gene [10]. The resulting male isoform of Imp (ImpM) cooperates with Bombyx mori Pelement somatic inhibitor (BmPSI) to promote malespecific splicing of $B m d s x$, which produces the male isoform of BmDSX (BmDSXM) [11, 12]. It has been recently reported that a long noncoding RNA transcribed from the Bmdsx-AS1 gene (designated as AS1 lincRNA) and the RNA binding protein BxRBP3A are also crucial for the male-specific splicing of $B m d s x[13,14]$. On the other hand, in individuals with the W chromosome, PIWI-interacting RNA (piRNA) is produced from the W-linked feminizer gene called Feminizer (Fem) [8]. The Fem piRNA-PIWI protein complex targets Masc mRNA for degradation. Insufficient levels of Masc expression result in the female-specific splicing of the Bmdsx premRNA and subsequent production of the female isoform of BmDSX (BmDSXF) [8]. Morphological analyses using transgenic silkworms that ectopically express either $B m d s x F$ or $B m d s x M$, as well as knockout silkworms homozygous for mutations in $B m d s x$, suggested that $B m d s x F$ and BmdsxM enhance female and male differentiation in gonads and external genitalia, respectively [15-18]. There have been no reports on the importance of $B m d s x$ in sexual differentiation in germ cells.

We previously demonstrated that transgenic expression of Fem-piRNA-resistant Masc gene (Masc-R) in females caused degenerated ovaries with testis-like tissues. Notably, the testis-like tissues produced a considerable number of sperms [9]. These findings strongly suggest that Masc could direct the differentiation of genetically female (ZW) germ cells into sperms. However, it remains unclear whether Masc directly induces spermatogenesis or if it promotes male differentiation in germ cells indirectly by inducing the expression of $B m d s x M$. To answer this question, it is necessary to clarify the importance of $B m d s x$ in sexual differentiation of germ cells.

Bmds $x$ is a Bombyx ortholog of doublesex ( $d s x)$ [8]. In most insect species, pre-mRNA from the $d s x$ gene undergoes sexual dimorphic alternative splicing to yield female- and male-specific isoforms ( $d s x F$ and $d s x M)$, which promote female and male differentiation, respectively, in somatic cells [15-20]. In the fruit fly Drosophila melanogaster, $d s x F$ is dispensable for female differentiation in germ cells, and factors that are expressed only in genetically female germ cells, such as a protein product of ovarian tumor (otu), as well as the germlinespecific isoforms of ovo and Sex-lethal, are essential for oogenesis [21-23]. Hence female differentiation of germ cells occurs even if the sex of the surrounding somatic cells is male. On the other hand, $d s x M$ expression in the surrounding somatic cells is required for the male differentiation of germ cells. The protein product of $d s x M$ in the surrounding somatic cells stimulates the JAK/STAT signaling pathway in germ cells, which plays an essential role in the initiation of spermatogenesis [21-23]. Complete spermatogenesis is achieved only when the sex of germ cells is male (XY) because multiple genes 
crucial for spermatogenesis are located on the $\mathrm{Y}$ chromosome [21-23]. These findings suggest that the role of $d s x$ in sexual differentiation in Drosophila differ between somatic cells and germ cells. However, it remains unclear whether this is also the case in other insect species.

In this study, we performed further analysis using the Masc- $R$ strain in combination with several $B m d s x$ knockout lines to elucidate whether Masc directly induces spermatogenesis or if it promotes male differentiation in germ cells indirectly by inducing the expression of BmdsxM. To this end, we performed genetic analyses in Masc- $R$ females homozygous for mutations in either $B m d s x M$ or BmdsxF. Furthermore, we performed RNA immunoprecipitation (RIP) analysis using an anti-MASC antibody to identify RNAs that interact with the MASC protein. The present study provides several lines of evidence that $B m d s x$ regulates sexual differentiation in germ cells in response to Masc expression and that the MASC protein interacts with AS1 lncRNA, which is known to be involved in the male-specific splicing of $B m d s x$ pre-mRNA.

\section{Results}

BmdsxM knockout in Masc- $R$ females fully restores ovary development

Our previous study using the Masc- $R$ strain suggested a potential role of Masc and Bmdsx in the sexual differentiation of the gonads and germ cells [9]. However, it remains unclear whether Masc directly induces spermatogenesis or promotes male differentiation in germ cells indirectly by inducing the expression of $B m d s x M$; the latter is supported by the fact that the ectopic expression of the Masc mRNA in Masc- $R$ females results in the expression of both BmdsxM and BmdsxF. Therefore, to eliminate the influence of $B m d s x M$ expression, we generated Masc- $R$ females that do not express $B m d s x M$ by crossing the Masc- $R$ strain with a BmdsxM deletion mutant strain established in this study (Additional files 5 and 6). The BmdsxM mutant strain had a 7-bp deletion in the $B m d s x$ exon 5 that encodes for the male-specific open reading frame (ORF), producing a truncated version of the BmDSXM protein (Additional file 5). RT-PCR analysis using primers that can amplify both $B m d s x F$ and $B m d s x M$ at the same time revealed that transgenic expression of Masc- $R$ reproducibly induced $B m d s x M$ expression in females irrespective of the $B m d s x$ genotype (Additional file 8). qRT-PCR analysis demonstrated that the homozygous mutation in BmdsxM abolished BmdsxM expression in the Masc- $R$ females, while it increased $B m d s x F$ expression by as much as seven-fold compared with Masc- $R$ females heterozygous for the $B m d s x M$ mutation (Fig. 1a and b). Morphological analysis of the internal genitalia confirmed that Masc- $R$ females heterozygous for the BmdsxM mutation (genetically the same as the Masc$R /+$ females) formed degenerated ovaries (Fig. 1e), consistent with what has been previously reported for Masc- $R$ females [9]. Testis-like tissues were observed at the apical end of ovarioles (Fig. 1h).

In comparison, Masc- $R$ females homozygous for the $B m d s x M$ mutation (thus only expressing $B m d s x F$ even in the presence of the Masc- $R$ gene) had fully developed ovaries as observed in normal females (Fig. If and g). Additionally, the morphological features of the apical end of ovarioles were similar to those observed in normal females (Fig. 1i and $\mathrm{j}$ ). No testis-like tissues were observed in Masc-R/+, Bmdsx ${ }^{M \Delta 7 / M \Delta 7}$ females. These results demonstrated that the Masc- $R$ transgene indirectly promotes the development of male gonads and the germ cells by inducing expression of Bmds $x M$.

\section{BmdsxM knockout in Masc- $R$ females restores the ability of egg production}

We next investigated the fertility of Masc- $R$ females homozygous for the BmdsxM mutation. As reported previously, Masc- $R$ expression in females caused a significant decrease in the number of mature eggs (Fig. 2a, Masc-R/+, Bmdsx $x^{M \Delta 7 /+}$ ) [9]. Interestingly, the homozygous mutation in $B m d s x M$ restored the number of mature eggs produced by Masc- $R$ females (Fig. 2a, Masc-R/+, Bmdsx ${ }^{M \Delta 7 / M \Delta 7}$ ). The hatchability of eggs laid by the Masc- $R$ females homozygous for the $B m d s x M$ mutation was also similar to that of normal females (Fig. 2b). These results demonstrated that BmdsxM knockout fully restored the ability of egg production in Masc- $R$ females.

\section{BmdsxM knockout in Masc- $R$ females induces the expression of genes essential for oogenesis}

To get more insight into the gene expression profile in the gonads of Masc-R/+, Bmdsx ${ }^{M \Delta 7 / M \Delta 7}$ females, we performed qRT-PCR to quantify expression levels of Bmovo-1 and Bm-nosO, both of which are important for oogenesis in the silkworm [24, 25]. While Masc- $R$ expression in females suppressed the expression of these two genes (Fig. 1c and 3d), the expression levels of Bmovo-1 and Bm-nosO were restored in Masc- $R$ females homozygous for the $B m d s x M$ mutation. These results further supported our previous findings that the egg production ability was restored in Masc-R females homozygous for the $B m d s x M$ mutation (Fig. 2a and b).

Combined, these results demonstrated that $B m d s x M$ depletion in Masc- $R$ females restored the ability of egg production by inducing the expression of genes important for oogenesis and egg formation. 

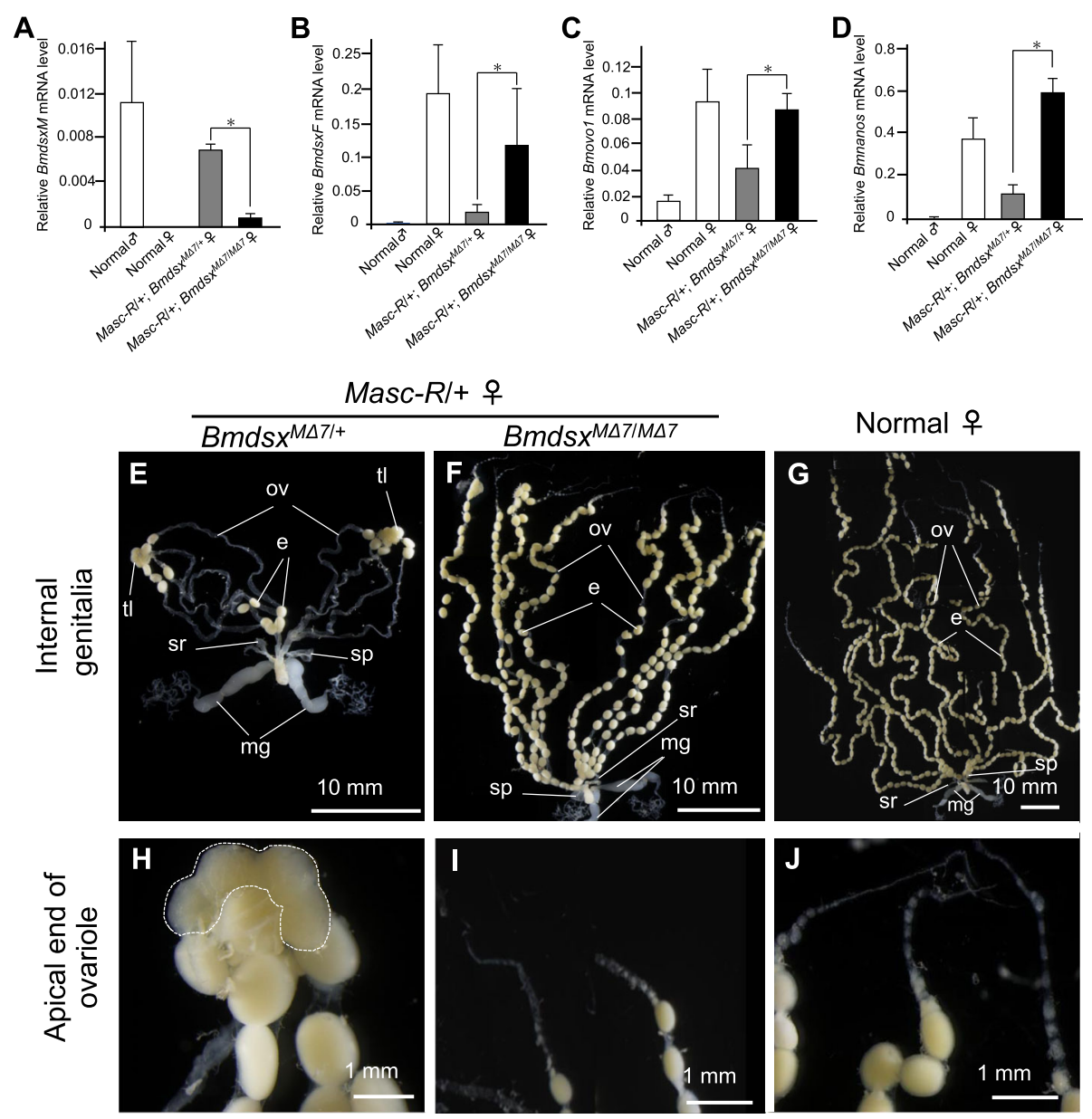

Fig. 1 Morphological and molecular analysis of the internal genitalia of Masc-R females homozygous for BmdsxM mutation. Homozygous mutation in $B m d s X M$ fully restored ovary development in Masc-R females. $\mathbf{a}$, $\mathbf{b}$ The expression levels of $B m d s \times M(\mathbf{a})$ and $B m d s \times F(\mathbf{b})$ in animals with the indicated genotype were quantified by qRT-PCR. c, d Expression of Bmovo-1 (c) and Bm-nosO (d), which is important for oogenesis, was quantified by qRT-PCR to evaluate the effect of $B m d s x M$ knockout on gonadal sexual development in Masc-R/+ females. Error bars indicate standard deviation. * indicates a significant difference, as determined by Welch's t-test. e- $\mathbf{g}$ Morphological analysis of the internal genitalia in Masc-R female heterozygous for $B m d s x^{M \Delta}{ }^{\prime}$ (E), Masc-R female homozygous for $B m d s x^{M \Delta T}(F)$, and normal female animals (g). e, egg; mg, mucous gland; ov, oviduct; sp., spermatheca; sr, seminal receptacle; $\mathrm{tl}$, testis-like tissue. $\mathbf{h}-\mathbf{j}$ High magnification images showing the apical end of the ovariole in Masc- $R$ female heterozygous for $B m d s X^{M \Delta 7} \mathbf{h}$, Masc- $R$ female homozygous for $B m d s x^{M \Delta\rangle}(I)$, and normal female animals $\mathbf{j}$. The dotted line indicates the testis-like tissues

BmdsxF depletion in Masc- $R$ females promotes female to male sex reversal in internal and external genitalia

We performed similar analyses using females with forced $M a s c-R$ expression and lack of BmdsxF expression, which were generated by crossing the Masc- $R$ strain with a BmdsxF deletion mutant strain that was established in this study (Additional files 6 and 10). This mutant strain had an 85-bp deletion in the female-specific $B m d s x$ exon (exon 3) and its adjacent intron sequence, resulting in no BmDSX protein production (Additional files 5 and 9). qRT-PCR analysis demonstrated that the homozygous mutation in BmdsxF abolished BmdsxF expression in the Masc- $R$ females. Although Masc- $R$ females expressed BmdsxM despite being female, BmdsxM expression levels were lower compared with Masc- $R$ females heterozygous for the BmdsxF mutation (Fig. 3a and b). Importantly, Masc- $R$ females homozygous for the $B m d s x F$ mutation developed internal genitalia that consisted of male-specific accessory glands, seminal vesicles, vas deferens, and ejaculatory duct and whose shape resembled normal male genitalia (Fig. 3f and g). In addition, testes similar in morphology with testes from normal males were observed at the apical end of the vas deferens in Masc-R/+, Bmdsx ${ }^{F \Delta 85 / F \Delta 85}$ females (Fig. 3i and $\mathrm{j}$ ). However, unlike normal males, the apical end of the vas deferens in these females was divided into several tubes (Fig. 3g and Additional file 12).

In comparison, Masc- $R$ females heterozygous for the $B m d s x F$ mutation (genetically the same as the Masc-R/+ females) formed degenerated ovaries (Fig. 3e) that were 

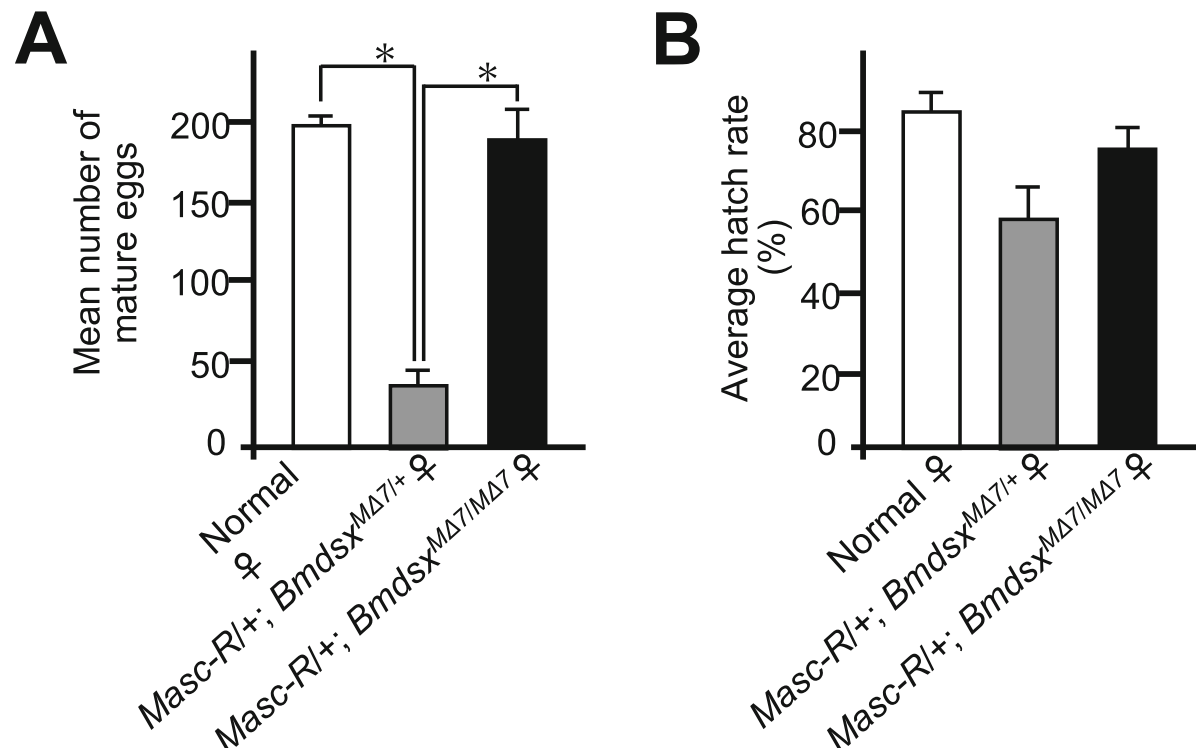

Fig. 2 Fertility of Masc-R females homozygous for BmdsxM mutation. Homozygous mutation in BmdsxM fully restored the fertility of Masc- $R$ females. $\mathbf{a}$ The mean number of mature eggs observed in adult ovaries. $\mathbf{b}$ Hatchability of eggs laid by females with the indicated genotype. Error bars indicate standard deviation. * indicates significant differences, as determined by Welch's $t$-test

consistent with those previously reported in Masc- $R$ females [9]. Moreover, testis-like tissues were observed at the apical end of ovarioles (Fig. 3h). These results indicated that BmdsxF depletion in Masc- $R$ females drives female to male sex reversal in the internal genitalia.

To extend our findings to other sexually dimorphic traits, we performed a morphological analysis of the external genitalia. Unlike similar previous studies of lepidopteran insects, we prepared cuticle specimens of the external genitalia, which enabled more accurate determination of the morphological changes in cuticle structures. The external genitalia of $M a s c-R /+$ females heterozygous for the BmdsxF mutation had morphological characteristics similar to those of normal females (Fig. 4a-6d). In comparison, the external genitalia of $M a s c-R$ females homozygous for the BmdsxF mutation were malformed, with partial development of several male-specific genital organs, such as the uncus, clasper, penis, and 9th tergite, which is unique to males (Fig. 4e and f). The shape of the ventral plate was also similar to that of normal males. These results strongly support our previous findings that $B m d s x F$ depletion in Masc- $R$ females promotes maleness.

\section{BmdsxF knockout in Masc- $R$ females enhances spermatogenesis}

We next investigated whether the testis-like tissues and the testes found in Masc- $R$ females heterozygous or homozygous for $B m d s x F$ mutation have the ability to produce spermatozoa. In the silkworm, males produce two types of sperm bundles, one of which consists of eupyrene sperm and the other of which is composed of apyrene sperm [27, 28]. The testis-like tissues observed in Masc- $R$ females heterozygous for the BmdsxF mutation contained sperm bundles that resembled apyrene sperm bundles (Fig. 5a and b). Similarly, the testes of $M a s c-R$ females homozygous for BmdsxF mutation contained apyrene sperm bundles (Fig. 5c). Although the testis-like tissues also contained sperm bundles that represented eupyrene sperm bundles, their size was smaller than that produced by normal male animals, and their shape was abnormal (Fig. $5 \mathrm{~d}$, e, and g). The testes of $M a s c-R$ females homozygous for BmdsxF mutation contained sperm bundles that resembled eupyrene sperm bundles, the size and shape of which were similar to those observed in male animals (Fig. $5 \mathrm{~d}, \mathrm{f}$, and g). These results demonstrated that $B m d s x F$ depletion, and thus the expression of Bmds $x M$ alone, promoted spermatogenesis, although the genetic sex of germ cells were all ZW females.

\section{BmdsxF knockout in Masc- $R$ females induces the expression of genes involved in spermatogenesis}

To evaluate the spermatogenesis observed in Masc-R/+, $B m d s x^{F \Delta 85 / F \Delta 85}$ females, we performed $\mathrm{qRT}-\mathrm{PCR}$ to quantify the expression of Bombyx orthologs of the Maelstrom (Mael) and always early (aly) genes (designated BmMael and BmAly, respectively), which are important for spermatogenesis and meiotic progression and spermatid differentiation in the silkworm $[29,30]$. Although the expression of these two genes in Masc- $R$ females heterozygous for the $B m d s x F$ mutation 

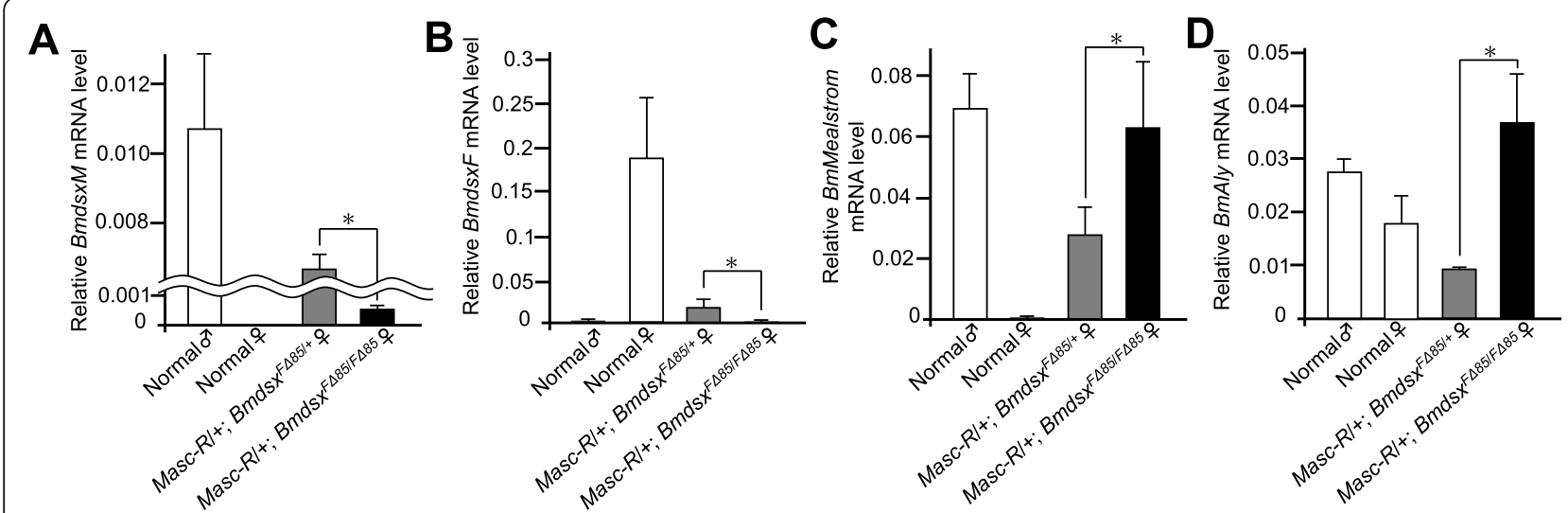

\section{Masc- $R /+$ 우}
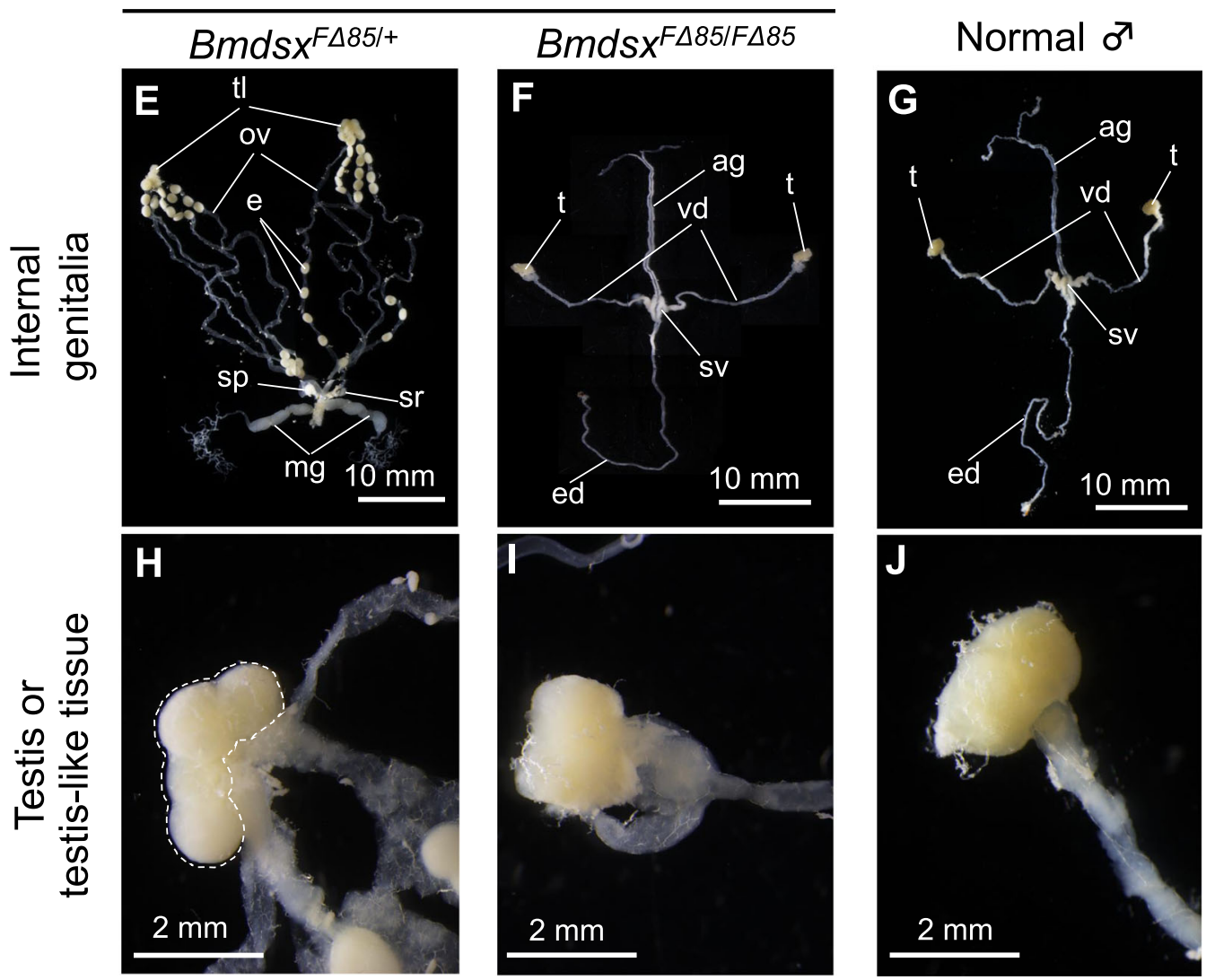

Fig. 3 Morphological and molecular analysis of the internal genitalia of Masc- $R$ females homozygous for Bmdsx $F$ mutation. Homozygous mutation in $B m d s x F$ promoted female to male sex reversal in the internal genitalia of Masc- $R$ females. (A, B) Expression levels of $B m d s \times M(\mathbf{a})$ and $B m d s x F(\mathbf{b})$ in animals with the indicated genotype were quantified by qRT-PCR. Expression levels of BmMaelstrom (c) and BmAly (d), both of which are involved in spermatogenesis, were also quantified by qRT-PCR to evaluate the effect of BmdsxF knockout on gonadal and germ cell sexual development in Masc$R /+$ females. Error bars indicate standard deviation. ${ }^{*}$ indicates a significant difference, as determined by Welch's $t$-test. e-g Morphological analysis of the internal genitalia in Masc-R female heterozygous for $B m d s x^{-\Delta 85}$ (e), Masc-R female homozygous for $B m d s x^{F \Delta 85}$ (f), and normal male animals (g). e, egg; mg, mucous gland; ov, oviduct; sp., spermatheca; sr, seminal receptacle; tl, testis-like tissue; ag, accessory gland; ed., ejaculatory duct; sv, seminal vesicle; t, testis; vd, vas deferens. $\mathbf{h}-\mathbf{j}$ High magnification images showing testis-like tissues and testes in Masc-R female heterozygous for Bmds ${ }^{F \Delta 85}$ (h), Masc- $R$ female homozygous for $B m d s x^{\triangleleft \Delta 85}$ (i), and normal male animals (j). The dotted line indicates the testis-like tissues

(genetically the same as Masc- $R /+$ females) was higher than that in normal females, the levels were still lower than those in normal males (Fig. $3 \mathrm{c}$ and $\mathrm{d}$ ). The expression of BmMaelstrom and BmAly in Masc-R females homozygous for the $B m d s x F$ mutation was significantly higher than in Masc- $R$ females heterozygous for 


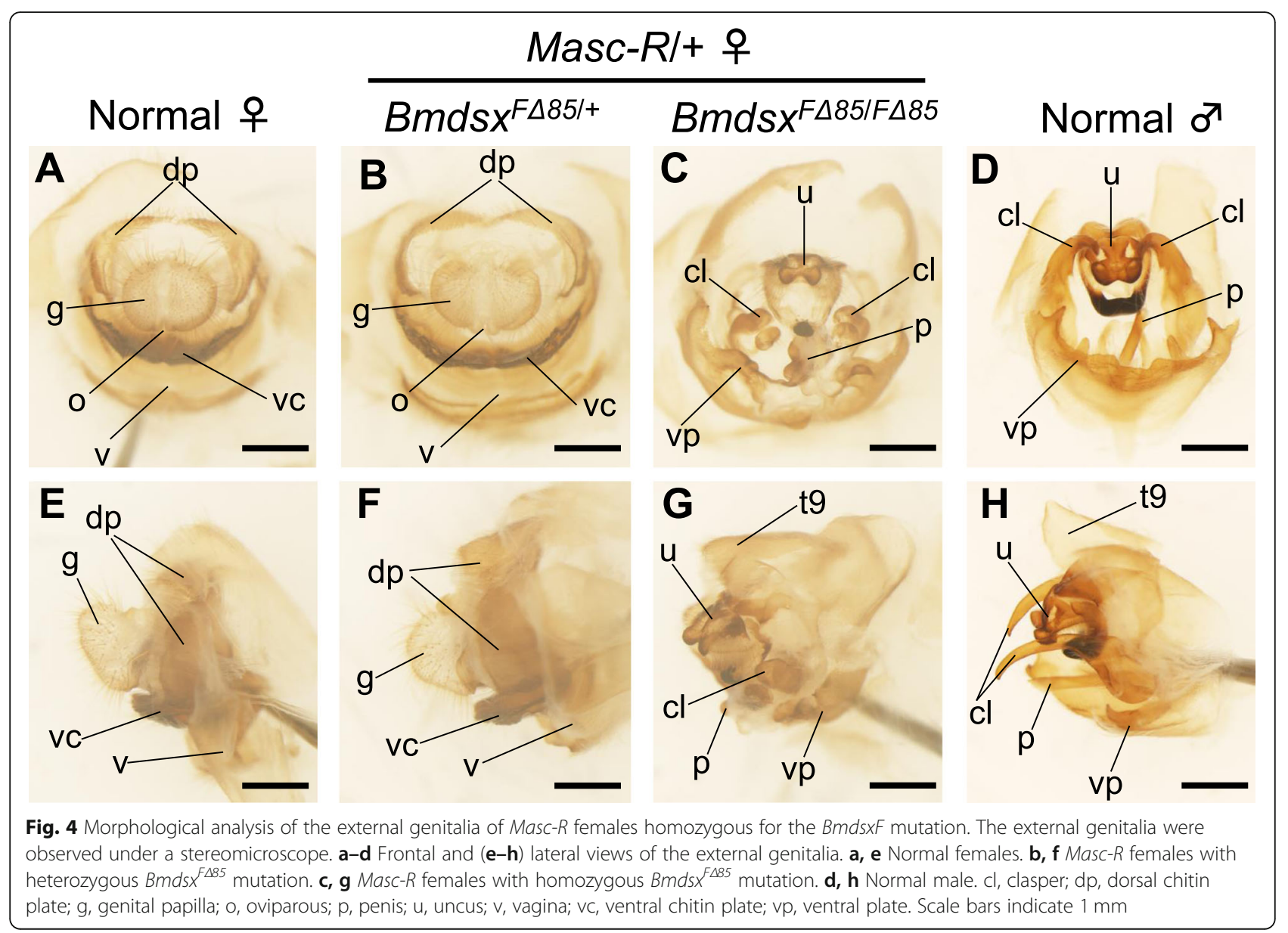

the $B m d s x F$ mutation and were comparable to those in normal males (Fig. $3 \mathrm{c}$ and d). These results support the above findings that $B m d s x F$ depletion in Masc-R females promotes spermatogenesis by increasing the expression of genes important for spermatogenesis (Fig. 4).

Combined, these results demonstrate that $B m d s x F$ depletion in Masc- $R$ females restored the ability to produce eggs by inducing the expression of genes important for spermatogenesis, meiotic progression, and spermatid differentiation.

\section{MASC protein interacts with the IncRNA from the Bmdsx- AS1 gene}

Our results revealed that Masc- $R$ promotes the development of male characteristics in genitalia, including the gonads and the germ cells, by inducing the expression of $B m d s x M$. Masc is required for the male-specific splicing of $B m d s x$ transcripts, giving rise to $B m d s x M[8,10]$. Therefore, we hypothesized that Masc might directly mediate male-specific Bmdsx splicing. To assess the potential interaction between Masc and Bmdsx, we performed RNA immunoprecipitation (RIP) in testis samples using a polyclonal antibody against MASC protein. Western blotting using whole protein extract from testes revealed that our anti-MASC antibody specifically recognized a protein with a molecular weight that was consistent with the putative molecular weight of the MASC protein $(64.8 \mathrm{kDa}$, Fig. 6a, left panel). The same Western blotting with an anti-DSX-DBD antibody detected a single protein band with the putative molecular weight of the BmDSXM $(30.0 \mathrm{kDa})$ and BmDSXF $(29.5 \mathrm{kDa})$ proteins (Fig. 6a, right panel). Immunostaining using the anti-MASC antibody indicated that MASC protein was predominantly localized in cells at the testicular basement membrane (Fig. 6b, e and i). Similarly, immunostaining analysis using the anti-DSX-DBD antibody revealed that the BmDSX protein was also expressed in cells of the testicular basement membrane (Fig. $6 \mathrm{f}$ and $\mathrm{j}$ ) and that it co-localized with MASC (Fig. $6 \mathrm{~g})$. These results were confirmed by in situ hybridization (ISH) using Masc or Bmdsx-specific riboprobes (Fig. 6c). Moreover, immunostaining demonstrated the co-localization of MASC and BmDSX in the cell nucleus (Fig. 6k, arrow heads).

The co-localization of MASC with BmDSX in the nucleus of cells found in the testicular basement membrane 


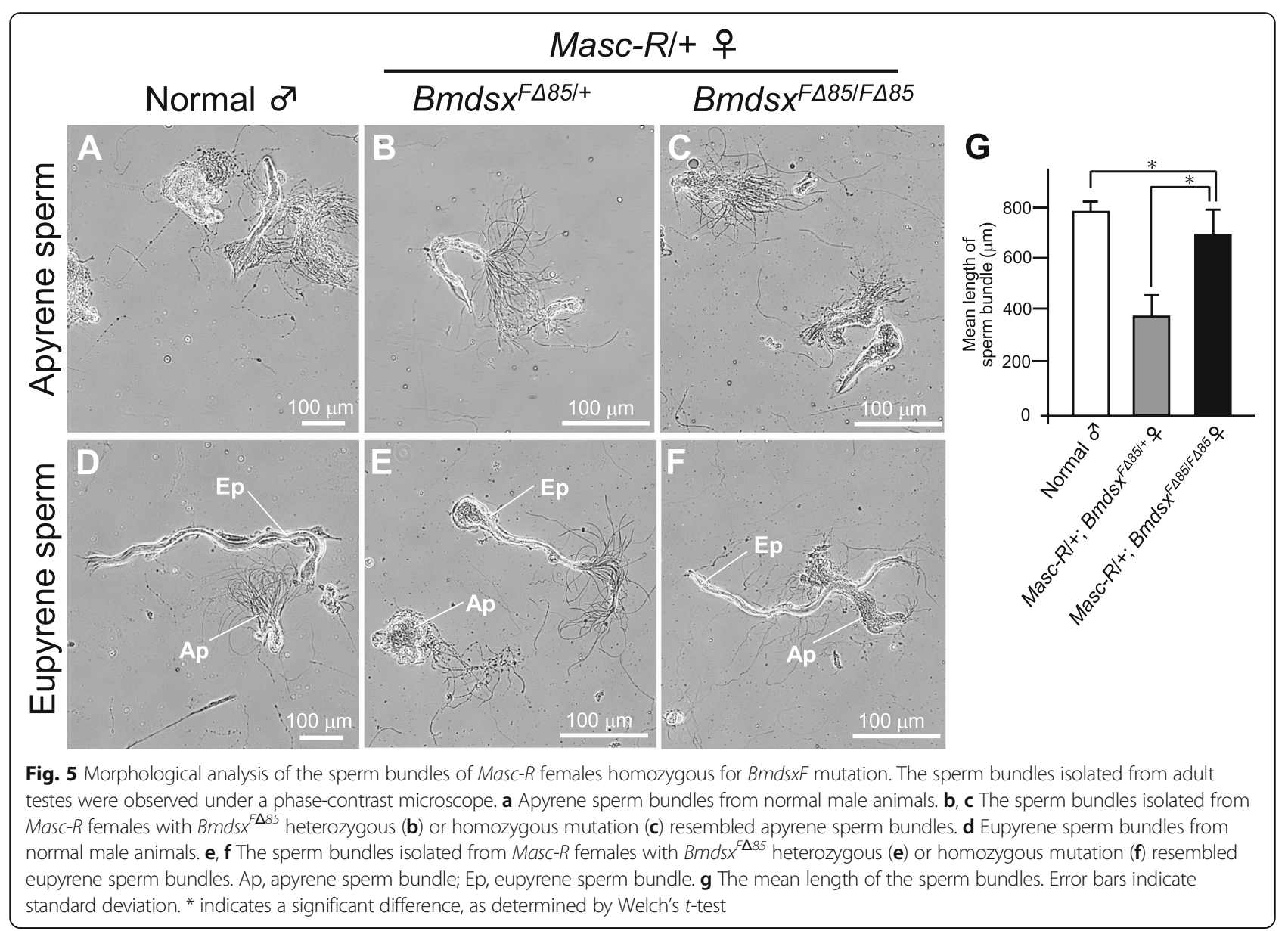

further supported the possibility that MASC may directly promote male-specific splicing in $B m d s x$. To investigate whether MASC interacts with Bmdsx pre-mRNA, we performed RIP using the anti-MASC antibody, followed by qRT-PCR. Contrary to our expectation, we found no significant enrichment of MASC on Bmdsx pre-mRNA (Fig. 6d). Instead, specific binding of MASC on a long noncoding RNA transcribed from the $B m d s x$ $A S 1$ gene (designated as AS1 lincRNA), which is a testisspecific factor involved in the male-specific splicing of $B m d s x$, was observed with the same analysis (Fig. 6d) [13]. Significant enrichment was not observed in any of the other RNAs examined, some of which were genes reportedly implicated in male-specific $B m d s x$ splicing. These results suggested the possibility that physical interaction between MASC and AS1 lncRNA may be important for inducing the male-specific splicing of $B m d s x$ pre-mRNA, giving rise to $B m d s x M$ in the testis.

\section{Discussion}

In this study, we clarified the genetic relationship between Masc and $d s x$ using a transgenic line and different mutant lines newly established by genome editing.
To our knowledge, few studies have revealed the genetic interactions between multiple genes with such genetic analysis in insects other than Drosophila. We showed that BmdsxM depletion in Masc-R female silkworms fully restored ovary development (Fig. If and i) and female fertility (Fig. 2a and b), while BmdsxF knockout caused female to male sex reversal in the internal genitalia of Masc- $R$ females (Fig. 3f and i). These results suggest that $B m d s x$ acts as a critical regulator of sexual differentiation in the internal and external genitalia of the silkworm, including the gonads and germ cells. Moreover, Masc acts as an intermediate regulator that shifts the sexual differentiation of the internal genitalia from female to male by promoting malespecific splicing in $B m d s x$ pre-mRNA. Although several studies have reported the functions of Masc and $d s x$ in lepidopterans, they have only partly captured the effects of these two genes on sexually dimorphic traits. Our study is the first to elucidate comprehensively the role of Masc and Bmdsx in the sexual differentiation of a wide range of sexually dimorphic traits including germ cells, gonads, internal reproductive organs, and the external genitalia. 


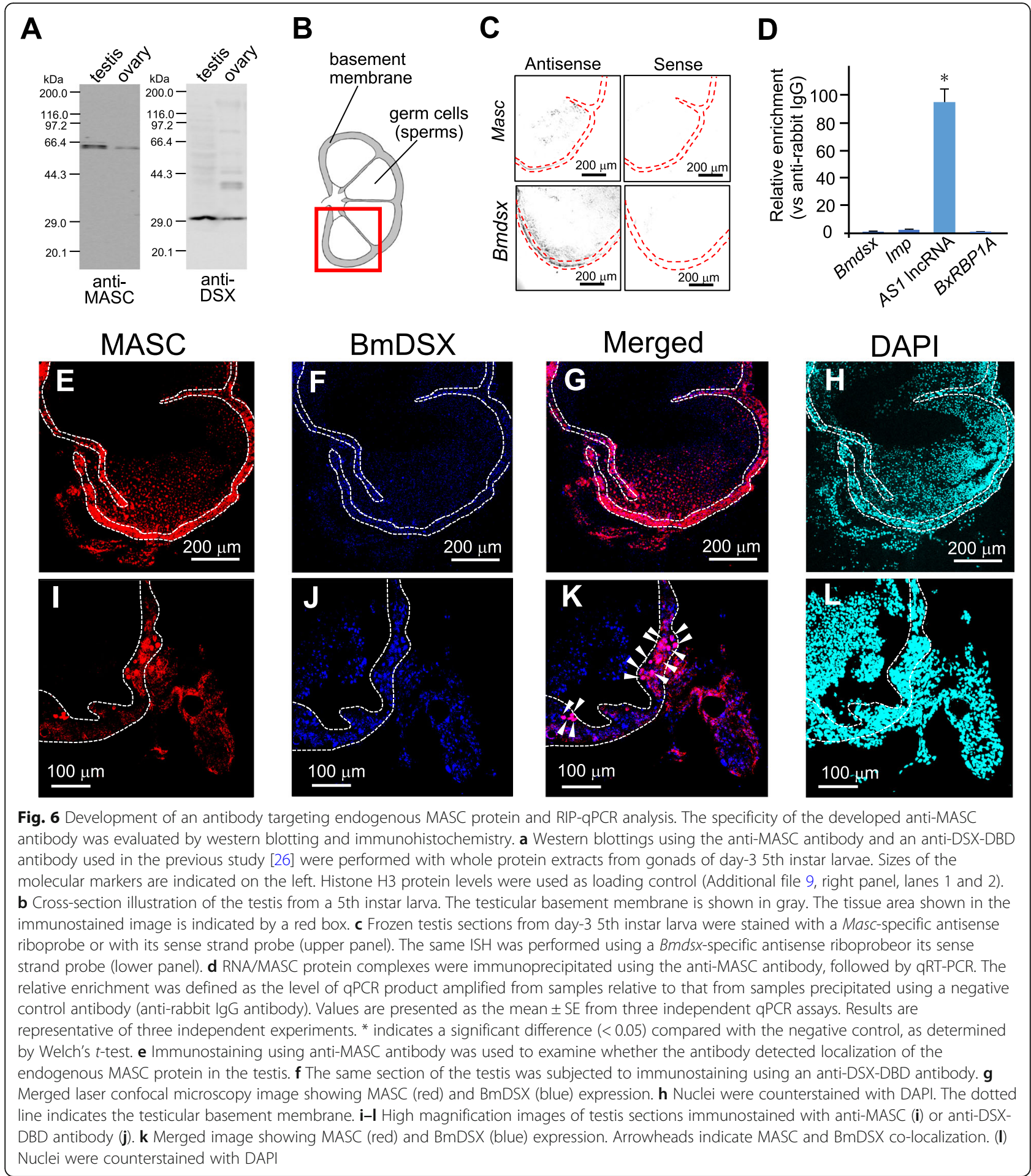

The expression of $B m d s x F$ in $M a s c-R$ females homozygous for the $B m d s x M$ mutation was significantly higher by seven-fold compared with Masc- $R$ females heterozygous for the $B m d s x M$ mutation. This is likely because the 7-bp deletion introduced in $B m d s x\left(B m d s x^{M \Delta 7}\right)$ had some effect on the sex-specific splicing of $B m d s x$ premRNA. While $B m d s x$ undergoes female-specific splicing by default [31], the $B m d s x^{M \Delta 7}$ mutation may have impaired the efficiency of male-specific $B m d s x$ splicing, resulting in increased levels of BmdsxF mRNA production. This hypothesis is further supported by the finding that increased levels of $B m d s x F$ expression were also observed in males homozygous for the $B m d s x^{M \Delta 7}$ mutation (Additional file 7). 
In addition to $B m d s x F$, the expression of Bmovo- 1 and $B m-n o s O$ was significantly increased in Masc- $R$ females homozygous for the $B m d s x^{M \Delta 7}$ mutation (Fig. 1c and d). These results suggest that $B m d s x F$ positively regulates Bmovo-1 and Bm-nosO expression, while BmdsxM suppresses their expression. Bmovo-1 protein is primarily found in the nuclei of ovarian cells and has been implicated in oogenesis. Bmovo-1 knockdown resulted in degenerated ovaries and markedly fewer oocytes [24]. $\mathrm{Bm}$-nosO is required for the arrangement of eggs in the ovariole and normal morphogenesis of eggs [25]. BmDSX proteins act as transcription factors regulating the expression of genes involved in the development of sexually dimorphic traits [15]. Consistent with previous findings, our immunostaining analysis using an antiDSX antibody revealed the nuclear localization of BmDSX proteins (Fig. 6f and j). BmDSXF likely functions as a transcription factor to induce the expression of Bmovo-1 and Bm-nosO, promoting egg development. There are no reports that $d s x$ is involved in the regulation of ovo and nanos in other insect species, including Drosophila. Therefore, it is reasonable to consider that $B m d s x$ controls oogenesis through a pathway unique to the silkworm. Further studies should assess whether BmDSXF directly regulates the transcription of Bmovo-1 and $B m-n o s O$.

The expression of BmdsxM in Masc- $R$ females homozygous for the $B m d s x F$ mutation was considerably lower compared with Masc- $R$ females heterozygous for the same mutation (Fig. 3a). This reduction in $B m d s x M$ expression in $B m d s x^{F \Delta 85 / \Delta 85}$ females may have resulted from the 85-bp deletion $\left(B m d s x^{F \Delta 85}\right)$ in the femalespecific alternative Bmdsx exon (exon 3 ) and its adjacent intronic sequence (Additional file 5). Intronic sequences flanking alternatively spliced exons function as intronic splicing enhancers or silencers (ISE or ISS), which enhance or repress the splice-site decision, respectively [32-34]. The 85-bp deletion may have affected the efficiency of male-specific splicing of $B m d s x$ pre-mRNA, leading to reduced levels of $B m d s x M$ transcripts.

Despite the profoundly reduced $B m d s x M$ expression levels, Masc- $R$ females homozygous for the $B m d s x^{F \Delta 85}$ mutation developed internal genitalia whose morphology resembled normal male internal genitalia (Fig. 3f and g). The same homozygous mutation also caused a marked decrement in Bmdsx expression levels in males (Additional file 11); however, these animals did not show any abnormalities in genitalia and exhibited normal male fertility. It is reasonable to speculate that the expression levels of BmdsxM were sufficient to promote male development in the internal genitalia. Alternatively, loss of $B m d s x F$ expression may have a higher effect on normal male development. As shown in Fig. 3b, the expression levels of BmdsxF in Masc-R females homozygous for the
$B m d s x^{F \Delta 85}$ mutation were significantly lower compared with heterozygous females and similar to the expression levels observed in normal males. It has been reported that BmDSXF and BmDSXM compete with each other for target site binding when both are present [15]. In $D$. melanogaster, the final phenotype of male-specific morphological structures in males carrying the hsp83-dsxF transgene depended upon the ratio of DSXF and DSXM [35]. Thus, male development due to $B m d s x F$ depletion would be more profound in Masc-R females homozygous for $B m d s x^{F \Delta 85}$ mutation compared with heterozygous females.

In addition to the masculinization observed in the internal genitalia, the sperm in Masc- $R$ females homozygous for the $B m d s x^{F \Delta 85}$ mutation resembled normal male sperm morphologically to a greater extent than the sperm observed in females heterozygous for the same mutation (Fig. 5a-f). This was particularly remarkable for eupyrene sperm (Fig. 5f). These results are consistent with the finding that the expression of BmMael and BmAly in Masc- $R$ females homozygous for BmdsxF mutation was significantly higher than in the Masc- $R$ females heterozygous for the $B m d s x F$ mutation (Fig. 3c and d). BmMael, which is a Bombyx ortholog of Maelstrom, regulates spermatogenesis in the silkworm [29]. Maelstrom is also essential for spermatogenesis in Drosophila and the mouse $[36,37]$. The D. melanogaster always early gene (DmAly) is required for the onset of spermatid differentiation and maintenance of normal chromatin structure in primary spermatocytes [38, 39]. BmAly is a Bombyx ortholog of the DmAly gene and is important for meiotic progression and spermatid differentiation in the silkworm [30]. As is the case for the internal genitalia, the impact of $B m d s x F$ depletion might be more important for male differentiation of germ cells than the presence of sufficient $B m d s x M$ levels. It is possible that normal spermatogenesis is repressed in the presence of $B m d s x F$ transcripts. There are no reports that loss of function of the female-specific isoform of $d s x$ causes spermatogenesis in genetically female germ cells. This strongly suggests that $d s x$ in the silkworm has a novel function different from that of Drosophila $d s x$.

However, the masculinization of several features in the genitalia and germ cells was still incomplete. Unlike wild-type males, the apical end of the vas deferens observed in the Masc- $R$ females homozygous for BmdsxF mutation was divided into several tubes (Fig. 3i). Often the number of malformed tubes was four, which is consistent with the number of oviducts typically found in each ovary (Additional file 12). Thus, female to male sex reversal may have been incomplete in the apical end of the vas deferens, resulting in the formation of malformed tubes. Similar signs of incomplete male development were also observed in the external genitalia of 
Masc- $R$ females homozygous for BmdsxF mutation, where partial formation of male genital organs, such as uncus and clasper, was observed, in addition to the presence of female genital organs (Fig. $4 \mathrm{c}$ and g). These intersexual phenotypes may have resulted from insufficient levels of $B m d s x M$ expression. It is likely that male differentiation of the external genitalia is more dependent on BmdsxM than the internal genitalia.

Similar incomplete masculinization was also observed in sperm. The size of the eupyrene sperm bundles observed in Masc- $R$ females homozygous for the $B m d s x^{F \Delta 85}$ mutation was significantly smaller compared with wild-type male silkworms (Fig. 5f). The total number of sperm bundles appeared considerably lower (data not shown). These findings suggest that Masc- $R$ females homozygous for the $B m d s x^{F \Delta 85}$ mutation still lack certain factors vital for spermatogenesis. In D. melanogaster, in addition to sex determination signals from somatic cells, the sex chromosome constitution of the germ cells is important for germline sex determination. The presence of the $\mathrm{Y}$ chromosome is indispensable for spermatogenesis, as it contains certain genes that are essential for spermatogenesis. Similarly, the male chromosomal constitution (ZZ) may also be necessary for spermatogenesis in the silkworm. A significant number of testis-specific genes have already been mapped on the $\mathrm{Z}$ chromosome in the silkworm [40]. Such enrichment of testis-specifically expressed genes on the $\mathrm{Z}$ chromosome is closely related to the male-specific chromosomal constitution, where the male sex is determined by the two $\mathrm{Z}$ chromosomes [40]. Moreover, the expression of genes mapped on the $\mathrm{Z}$ chromosome in the testis was more than ten-fold higher on average than in the ovary [41]. Therefore, it is reasonable to speculate that the male chromosomal constitution (two $\mathrm{Z}$ chromosomes) in germ cells is essential for achieving complete spermatogenesis.

To our knowledge, this is the first study to demonstrate the interaction of MASC proteins with AS1 lncRNA (Fig. 6d). Immunostaining analysis demonstrated that MASC was localized in the nucleus of cells found on the testicular basement membrane (Fig. 6e and i). This result is consistent with previous studies reporting that MASC contains a nuclear localization signal and exhibits nuclear localization in cultured cells expressing a Masc transgene [42]. AS1 lncRNA expression was also observed in the nucleus of testicular cells [13]. These findings strongly suggest that MASC proteins bind to AS1 lncRNA in the cell nucleus. The gene encoding AS1 lncRNA is expressed in a testis-specific manner, and its knockdown in males shifts the splicing pattern of $B m d s x$ from male to female mode [13]. AS1 lncRNA contains a nucleotide sequence complementary to $B m d s x$ premRNA; hence, it is possible that $A S 1$ lncRNA interacts directly with $B m d s x$ pre-mRNA, inducing male-specific splicing. MASC proteins potentially interact with AS1 lncRNA, augmenting its ability to induce male-specific $B m d s x$ splicing. Since no significant enrichment of MASC on Bmdsx pre-mRNA was observed (Fig. 6d), it is conceivable that MASC-AS1 lincRNA interaction indirectly regulates the male-specific splicing of $B m d s x$ pre-mRNA. For example, MASC binding to AS1 lncRNA could protect the lncRNA from degradation, resulting in enhanced AS1 lncRNA-mediated male-specific Bmdsx splicing. How exactly the interaction between MASC and AS1 lncRNA promotes male-specific splicing in $B m d s x$ pre-mRNA remains to be elucidated.

Our Western blotting analysis unexpectedly detected the weak but definite MASC expression in the ovary (Fig. 6a). In this regard, it has recently been reported that Masc has an important role in female differentiation of the external genitalia [43]. This suggests that Masc has some functions in female differentiation. Our study is the first example to suggest that Masc may have some functions for the female differentiation in the internal genitalia and or germ cells. This finding will be a great help to understand the novel features of Masc.

\section{Methods}

\section{Silkworm maintenance, generation of Bmdsx knockout silkworms, and crossing}

Bombyx mori strains were maintained under standard conditions [44]. The Masc- $R$ strain, the generation of which was described in our previous study [9], was used in the present study. Knockout silkworms were generated using transcription activator-like effector nucleases (TALENs), as described previously [45, 46]. The target sites of TALENs within the female-specific exon (exon 3 ) and male-specific coding region in exon 5 are illustrated in Additional file 5. Generation 0 (G0) hatched larvae were reared to adults, and G0 adults were crossed. Mutant strains were established and maintained as described in our previous study [46]. Primer sequences and PCR conditions used for genotyping are shown in Additional file 1. Genomic PCR for genotyping was performed according to a previously described protocol [47]. The resulting mutant lines $B m d s x^{F \Delta 85}$ and $B m d s x^{M \Delta 7}$ harbored an 85 -bp deletion and a 7-bp deletion within the target sites, respectively (Additional file 5). Masc- $R /+$ males were bred with females homozygous for the $B m d s x^{M \Delta 7}$ mutation, and Masc-R/+ females homozygous for the $B m d s x^{M \Delta 7}$ mutation were obtained according to the procedure illustrated in Additional file 6 . Masc-R/+ females homozygous for the $B m d s x^{F \Delta 85}$ mutation were generated following the procedure described in Additional file 10. Wild-type females and males obtained from the same parent were served as normal female and male controls. 
RNA extraction and reverse transcription (RT)-PCR

Total RNA extraction from silkworm tissues using ISOGEN (Nippon Gene) and subsequent RT-PCR was performed as described previously [48]. The primer sequences and PCR conditions used for the RT-PCRs are shown in Additional file 2.

\section{Quantitative real-time RT-PCR (qRT-PCR)}

qRT-PCR assays were performed according to a previously described protocol [45]. The primer sequences used for qRT-PCR are listed in Additional file 3. Amplification of elongation factor-2 (EF-2) as an internal control using the BmEF-2F1 and BmEF-2R1 primers was also performed for quantification [49].

\section{In situ hybridization (ISH)}

Localization analysis of Masc and Bmdsx mRNAs using in situ hybridization (ISH) was performed as previously described [50, 51]. Digoxygenin-labeled sense and antisense riboprobes were synthesized using a DIG RNA labeling kit (Roche) and PCR-amplified cDNAs as a template, according to the manufacturer's instructions. The PCR primer sequences used for ISH are listed in Additional file 4.

\section{Western blotting}

Testes and ovaries from day-3 5 th instar larvae were sonicated (two sets of 1 -s pulse $\times 10$ times) in $2 \times$ sample buffer (100 mM Tris-HCl [pH 6.8], 4\% SDS, $12 \% \beta$ mercaptoethanol), followed by incubation at $95^{\circ} \mathrm{C}$ for 3 $\mathrm{min}$. The resulting product was centrifuged $(15,000 \mathrm{rpm}$, $\left.25^{\circ} \mathrm{C}, 5 \mathrm{~min}\right)$, and the supernatants were used for western blotting. Equal amounts $(5 \mu \mathrm{g})$ of protein were separated by $12.5 \%$ SDS-PAGE and then transferred onto PVDF membranes. The membranes were immunoblotted with an anti-MASC polyclonal antibody (1:50 dilution) or an anti-DSX-DBD antibody (1:50 dilution) in blocking buffer ( $1 \times$ TBS-T containing $5 \%$ skimmed milk powder) overnight at $4{ }^{\circ} \mathrm{C}$. The anti-MASC antibody was produced by Eurofins Genomics. The C-terminal fragment of MASC (CASKERKPEARNTEI), which was predicted to be the most appropriate epitope, was synthesized and used to immunize a rabbit. The antiDSX-DBD monoclonal antibody was purchased from Developmental Studies Hybridoma Bank. HRPconjugated mouse anti-rabbit IgG (1:3000; sc-2357, Santa Cruz Biotechnology) and an HRP-conjugated goat anti-mouse IgG-HRP (1:3000; sc-2005, Santa Cruz Biotechnology) were used as secondary antibodies. Using a detection reagent (Immunostar LD, Wako), the chemiluminescence signal on the membranes was detected on an ImageQuant LAS4000 system (GE Healthcare).

\section{Immunohistochemistry}

Frozen sections were prepared using a cryostat and then fixed with acetone at $-20^{\circ} \mathrm{C}$. The sections were washed three times for 5 min with $1 \times \mathrm{TBS}(\mathrm{pH} 7.5)$, and then incubated in blocking buffer ( $1 \times$ TBS-T containing $5 \%$ normal goat serum) for $1 \mathrm{~h}$ at room temperature. After blocking, the sections were incubated either with antiMASC antibody (1:10 dilution) or anti-DSX-DBD antibody (1:10 dilution) overnight at $4{ }^{\circ} \mathrm{C}$. After three washes with $1 \times$ TBS (pH 7.5) for 5 min, the sections were incubated with a secondary antibody according to the protocol described previously [52]. Fluorescence images were acquired using the confocal laser scanning microscope FV3000 (Olympus).

\section{RNA Immunoprecipitation qPCR (RIP-qPCR)}

The RIP-qPCR analysis was performed according to a previously described protocol with minor modifications [53]. Briefly, RIP samples were prepared from ten pooled testes of day-3 5th instar larvae using the anti-MASC antibody (12 $\mu$ g per reaction). RNAs were extracted from the RIP samples using ISOGEN followed by treatment with TURBO DNase (Thermo Fisher Scientific), according to the manufacturer's instructions. To calculate the amount of target sequence in the precipitated RNA/protein complexes, we performed qRT-PCR, as previously described [53]. The primer sequences used for RIPqPCR are shown in Additional file 3.

\section{Supplementary information}

Supplementary information accompanies this paper at https://doi.org/10. 1186/s12861-020-00224-2.

Additional file 1: Table S1. Primer sequences and PCR conditions used for genotyping.

Additional file 2: Table S2. Primer sequences and PCR conditions used for RT-PCR.

Additional file 3: Table S3. Sequences of primers used for qRT-PCR. Additional file 4: Table S4. Sequences of primers used to prepare riboprobes for $\mathrm{ISH}$.

Additional file 5: Fig. S1. Generation of $B m d s x M$ and $B m d s x F$ knockout silkworm lines using TALENs. (A) The target sites of TALENs within the female-specific exon (exon 3 ) and the male-specific coding region in exon 5 are shown. The rectangles indicate exons. Exons 3 and 4 are skipped when Bmdsx pre-mRNA is spliced in males. The gray region encodes the female-specific open reading frame (ORF). The black region encodes the male-specific ORF. TAL effector-binding sequences are shown in blue, while spacer sequences are indicated in red. (B) The deletion mutations introduced in the $B m d s x^{F \Delta 85}$ and $B m d s x^{M \Delta 7}$ lines are shown in (B) and $(C)$, respectively. The uppercase characters in (B) represent the nucleotide sequence of Bmdsx exon 3. The uppercase characters in (C) indicate the nucleotide sequence of Bmdsx exon 5. Colons indicate identical nucleotide sequences between wild-type and mutant animals. Spacer sequences are indicated in red. 5' splice donor and $3^{\prime}$ splice acceptor sites are shown in bold characters.

Additional file 6: Fig. S2. Procedure followed to generate Masc- $R /+$ females homozygous for the $B m d s x^{M \Delta 7}$ mutation. The Masc- $R /+$ females homozygous for Bmds ${ }^{M \Delta 7}$ were generated by crossing Masc- $R$ and $B$ Bmds $X^{M \Delta 7}$ animals. (A) In generation $0(\mathrm{G} 0)$, Masc- $R /+$ males were crossed 
with females homozygous for the Bmds ${ }^{M \Delta>}$ mutation. In the nex generation (G1), animals heterozygous for the $B m d s x^{M \Delta 7}$ mutation were selected after PCR-based genotyping, and females without the Masc- $R$ transgene were crossed with Masc-R/+ males. Individuals carrying the Masc $-R$ transgene were selected based on the expression of the egfp marker gene, as described previously [9]. In the resulting offspring (G2), Masc-R/+ females homozygous for the $B m d s x^{M \Delta}{ }^{7}$ mutation were subjected to further analyses. Individuals heterozygous for the $B m d s x^{M \Delta}{ }^{\text {> }}$ mutation or individuals with wild-type BmdsXM were used as controls. (B) PCR-based genotyping for the identification of individuals homozygous or heterozygous for the Bmdsx ${ }^{M \Delta 7}$ mutation. Genomic PCR was performed as described in Materials and Methods, and the amplified product was separated by $10 \%$ polyacrylamide gel electrophoresis. The gels were stained with $1 \%$ ethidium bromide in $1 \times$ TAE buffer to visualize the DNA. The upper bands represent amplicons from wild-type Bmdsx animals, while the lower bands represent amplicons from $B m d s x^{M \Delta 7}$ mutants.

Additional file 7: Fig. S3. Bmdsx mRNA levels in $B m d s x^{E \Delta 85}$ and $B m d s x^{M \Delta 7}$ silkworms. Expression levels of $B m d s x$ at the mRNA level in the $B m d s x$ mutant lines used in this study were analyzed by qRT-PCR. $B m d s \times M$ mRNA levels in the internal genitalia of $B m d s x^{M \Delta>}(A)$ and $B$ mds $x^{-\triangle 85}$ mutant animals (C), as determined by qRT-PCR. Similarly, the mRNA level of $B m d s x F$ was quantified by qRT-PCR in $B m d s x^{M \Delta\rangle}(B)$ and $B m d s x^{F \Delta 85}$ mutants (D). Error bars indicate standard deviation. * indicates a significant difference, as determined by Welch's $t$-test.

Additional file 8: Fig. S4. Expression pattern of $B m d s x$ was analyzed by RT-PCR using primers that can amplify both BmdsxF and BmdsxM transcripts at the same time. Template CDNAs were prepared from the internal genitalia of adults with indicated genotypes. The amplified product was separated by $10 \%$ polyacrylamide gel electrophoresis. The gels were stained with $1 \%$ ethidium bromide in $1 \times$ TAE buffer to visualize the DNA The arrows indicate the DNA bands corresponding to the size of $B m d s x F$, $B m d s \times M$, and $B m d s x^{F \Delta 85}$ transcripts.

Additional file 9: Fig. S5. BmDSX protein levels in $B m d s x^{\circledR \Delta 85}$ and $B m d s X^{M{ }^{7}}$ lines. BmDSX protein levels were determined by western blotting using an anti-DSX-DBD antibody (left panel). Whole protein extracts from testes or ovaries of day-3 5 th instar larvae with the indicated genotype were separated by $12.5 \%$ SDS-PAGE. The sizes of the molecular markers are indicated on the left. The arrow indicates the protein band corresponding to the molecular weight of each BmDSX protein. The expected molecular weights were as follows: BmDSXM, $32 \mathrm{kDa}$; BmDSXF, $29.5 \mathrm{kDa} ; \mathrm{BmDSX}^{\mathrm{M} \Delta 7}$, $26.6 \mathrm{kDa}$; BmDSX$X^{\mp \Delta 85}$, $24.8 \mathrm{kDa}$. Histone $\mathrm{H3}$ protein levels were used as loading control (right panel).

Additional file 10: Fig. S6. Procedure followed to generate Masc-R/+ females homozygous for the $B m d s x{ }^{\Delta 85}$ mutation. Masc-R/+ females homozygous for $B m d s x^{F \Delta 85}$ were generated by crossing Masc $-R$ and $B m d s x^{F \Delta 85}$ animals. (A) In generation 0 (GO), Masc-R/t+ females were crossed with males homozygous for the $B m d s x^{x^{-\Delta 85}}$ mutation. In the next generation (G1), animals heterozygous for the $B m d s x^{F \Delta 85}$ mutation were selected after PCR-based genotyping, and females without the Masc- $R$ transgene were crossed with Masc-R/+ males. Individuals carrying the Masc- $R$ transgene were selected based on the expression of the egfp marker gene, as described previously [9]. In the resulting offspring (G2), Masc-R/+ females homozygous for the $B m d s x^{E \Delta 85}$ mutation were subjected to further analyses. Individuals heterozygous for the $B m d s x^{F \Delta 85}$ mutation or individuals with wild-type BmdsXF were used as controls. (B) PCR-based genotyping for the identification of individuals homozygous or heterozygous for the Bmdsx $x^{\mp \Delta 85}$ mutation. Genomic PCR was performed as described in Materials and Methods, and the amplified product was separated by $2 \%$ agarose gel electrophoresis. The gels were stained with $1 \%$ ethidium bromide in $1 \times$ TAE buffer to visualize the DNA. The upper bands represent amplicons from wildtype Bmdsx, while the lower bands represent amplicons derived from $B m d s x^{\mp \Delta 85}$ animals.

Additional file 11: Fig. S7. Expression levels of $B m d s \times M$ in males homozygous for the $B m d s x^{F \Delta 85}$ mutation. Expression levels of $B m d s \times M$ mRNA in males homozygous for the Bmds $x^{\ulcorner\Delta 5}$ mutation were determined by qRT-PCR. Error bars represent standard deviation. * indicates a significant difference, as determined by Welch's $t$-test.
Additional file 12: Fig. S8. Malformed tubes observed at the apical end of the vas deferens in Masc-R females homozygous for the BmdsxF mutation. Images around the apical end of the vas deferens were acquired by a digital camera attached to a stereomicroscope. (A) Normal male. (B-E) Masc-R females homozygous for the Bmdsx ${ }^{F \Delta 85}$ mutation. The dotted lines indicate malformed tubes. T: testis, VD: vas deferens.

\section{Abbreviations}

Masc: Masculinizer; dsx: Doublesex; Bmdsx: Bombyx mori doublesex $B m d s x M$ : Male isoform of Bmdsx; BmdsxF: Female isoform of Bmdsx; RIP: RNA immunoprecipitation; IncRNA: Long non-coding RNA; ORF: Open reading frame; RT-PCR: RNA extraction and reverse transcription-PCR; qRTPCR: Quantitative real-time RT-PCR; Bmovo-1: Bombyx mori ovo-1; BmnosO: Bombyx mori nanos ortholog; BmMaelstrom: Bombyx mori Maelstrom; BmAly: Bombyx mori always early (Aly); Bmdsx-AS1: Bmdsx-anti-sense RNA 1; mRNA: Messenger RNA; SDS: Sodium dodecyl sulfate; PAGE: Polyacrylamide gel electrophoresis; PBS: Phosphate-buffered saline; TAE: Tris-acetate-EDTA buffer

\section{Acknowledgments}

We thank K. Nakamura and T. Misawa for maintaining silkworm strains. We are grateful to S. Kobayashi and M. Sakato for their excellent assistance with the experiments. We also thank A. Suzuki for drawing the illustrations used in the figures. We express our sincere gratitude to Dr. Yoko Takasu for her advice regarding TALEN scaffold construction.

\section{Authors' contributions}

TY performed all of the molecular biology experiments (RT-PCR, GRT-PCR, western blotting, and RIP-qPCR), histochemical experiments, and mutant fertility tests. TY and MM made the morphological observations. MM and MS established the BmdsxF and BmdsxM mutant strains. MGS analyzed the anatomy of the internal and external genitalia. TY, FA, HS, and MGS conceived and designed this work. All of the authors discussed the data and helped in preparation of the manuscript. TY and MGS wrote the manuscript with intellectual input from all authors. All authors read and approved the final manuscript.

\section{Funding}

This work was supported by Grants-in-Aid for Scientific Research (B) $17 \mathrm{H} 03940$ (to MGS) and 20H02996 (to MGS). This work was supported in part by Grant-inAid for Scientific Research (C) 17 K08161 (to MS). The funding bodies had no role in designing the study, collection, analysis, and interpretation of the data, or writing of the manuscript.

\section{Availability of data and materials}

All of the mutant lines established in this study are continuously reared and passaged at the Laboratory of Bio-resource Regulation, Department of Integrated Biosciences, Graduate School of Frontier Sciences, The University of Tokyo and Genetically Modified Organism Research Center, National Institute of Agrobiological Sciences. All data obtained or analysed during the present study are available from the corresponding author on reasonable request.

Ethics approval and consent to participate

Not applicable.

\section{Consent for publication}

Not applicable.

\section{Competing interests}

The authors declare that they have no competing interests.

\section{Author details}

${ }^{1}$ AIR WATER INC, 4-9-4 Hatchobori, Chuo-ku, Tokyo 104-0032, Japan.

${ }^{2}$ Department of Integrated Biosciences, Graduate School of Frontier Sciences, The University of Tokyo, 5-1-5 Kashiwanoha, Kashiwa-shi, Chiba 277-8562, Japan. ${ }^{3}$ SHINYUSHA, 1-12 Kanda Jimbocho, Chiyoda-ku, Tokyo 101-0051, Japan. ${ }^{4}$ Genetically Modified Organism Research Center, National Institute of Agrobiological Sciences, Owashi, Tsukuba 305-8634, Japan. 
Received: 1 June 2020 Accepted: 10 September 2020 Published online: 21 September 2020

\section{References}

1. Herpin A, Schartl M. Plasticity of gene-regulatory networks controlling sex determination: of masters, slaves, usual suspects, newcomers, and usurpators. EMBO Rep. 2015;16:1260-74.

2. Sinclair H, Berta P, Palmer S, Hawkins R, Griffiths L, Smith J, et al. A gene from the human sex-determining region encodes a protein with homology to a conserved DNA-binding motif. Nature. 1990;346:240-4.

3. Yano A, Guyomard R, Nicol B, Jouanno E, Quillet E, Klopp C, et al. An immune-related gene evolved into the master sex-determining gene in rainbow trout, Oncorhynchus mykiss. Curr Biol. 2012;22:1423-8.

4. Hall B, Sanjay B, Xiaofang J, Yumin Q, Vladimir T, Biedler J, et al. A male determining factor in the mosquito Aedes aegypti. Science. 2015;348:1268-70.

5. Krzywinska E, Dennison NJ, Lycett GJ, Krzywinski J. A maleness gene in the malaria mosquito Anopheles gambiae. Science. 2016;348:67-9.

6. Sharma A, Heinze D, Wu Y, Kohlbrenner T, Morilla I, Brunner C, et al. Male sex in houseflies is determined by $M d m d$, a paralog of the generic splice factor gene CWC22. Science. 2017;356:642-5.

7. Hashimoto $\mathrm{H}$. The role of the $\mathrm{W}$ chromosome for sex determination in the silkworm, Bombyx mori. Jpn J Genet. 1933;8:245-58.

8. Kiuchi T, Koga H, Kawamoto M, Shoji K, Sakai H, Arai Y, et al. A single female-specific piRNA is the primary determiner of sex in the silkworm. Nature. 2014;509:633-6.

9. Sakai H, Sumitani M, Chikami Y, Yahata K, Uchino K, Kiuchi T, et al. Transgenic expression of the piRNA-resistant Masculinizer gene induces female-specific lethality and partial female-to-male sex reversal in the silkworm, Bombyx mori. PLoS Genet. 2016;12:e1006203.

10. Sakai $H$, Sakaguchi H, Aoki F, Suzuki MG. Functional analysis of sexdetermination genes by gene silencing with LNA-DNA gapmers in the silkworm, Bombyx mori. Mech Dev. 2015;137:45-52.

11. Suzuki MG, Imanishi S, Dohmae N, Nishimura T, Shimada T, Matsumoto S. Establishment of a novel in vivo sex-specific splicing assay system to identify a trans-acting factor that negatively regulates splicing of Bombyx mori dsx female exons. Mol Cell Biol. 2008;28:333-43.

12. Suzuki MG, Imanishi S, Dohmae N, Asanuma M, Matsumoto S. Identification of a male-specific RNA binding protein that regulates sex-specific splicing of Bmdsx by increasing RNA binding activity of BmPSI. Mol Cell Biol. 2010;30: 5776-586.

13. $X u X$, Wang $K$, Zha $X$. An antisense IncRNA functions in alternative splicing of Bmdsx in the silkworm, Bombyx mori. Biochem Biophys Res Commun. 2019;516:639-44

14. Zheng ZZ, Sun X, Zhang B, Pu J, Jiang ZY, Li M, Fan YJ, Xu YZ. Alternative splicing regulation of doublesex gene by RNA-binding proteins in the silkworm Bombyx mori. RNA Biol. 2019:16:809-20.

15. Suzuki MG, Funaguma S, Kanda T, Tamura T, Shimada T. Analysis of the biological functions of a doublesex homologue in Bombyx mori. Dev Genes Evol. 2003;213:345-54

16. Suzuki MG, Funaguma S, Kanda T, Tamura T, Shimada T. Role of the male BmDSX protein in the sexual differentiation of Bombyx mori. Evol Dev. 2005; 7:58-68.

17. $X u$ J, Wang $Y$, Li Z, Ling $L$, Zeng $B$, James $A A$, et al. Transcription activatorlike effector nuclease (TALEN)-mediated female-specific sterility in the silkworm, Bombyx mori. Insect Mol Biol. 2014;23:800-7.

18. Xu J, Zhan S, Chen S, Zeng B, Li Z, James AA, et al. Sexually dimorphic traits in the silkworm, Bombyx mori, are regulated by doublesex. Insect Biochem Mol Biol. 2017:80:42-51.

19. Inoue K, Hoshijima K, Higuchi I, Sakamoto H, Shimura Y. Binding of the Drosophila transformer and transformer-2 proteins to the regulatory elements of doublesex primary transcript for sex-specific RNA processing. Proc Natl Acad Sci U S A. 1992;89:8092-6.

20. Raymond S, Shamu E, Shen M, Seifert J, Hirsch B, Hodgkin J, et al. Evidence for evolutionary conservation of sex-determining genes. Nature. 1998;391: 691-5.

21. Casper A, Van Doren M. The control of sexual identity in the Drosophila germline. Development. 2006;133:2783-91.

22. Hempel LU, Kalamegham R, Smith JE 3rd, Oliver B. Drosophila germline sex determination: integration of germline autonomous cues and somatic signals. Curr Top Dev Biol. 2008;83:109-50.
23. Murray M, Yang Y, Van M. Germ cell sex determination: a collaboration between soma and germline. Curr Opin Cell Biol. 2010;22:722-9.

24. Xue R, Hu X, Cao G, Huang M, Xue G, Qian Y, et al. Bmovo-1 regulates ovary size in the silkworm, Bombyx mori. PLoS One. 2014;9:e104928.

25. Nakao H, Takasu Y. Complexities in Bombyx germ cell formation process revealed by Bm-nosO (a Bombyx homolog of nanos) knockout. Dev Biol. 2019:445:29-36.

26. Prakash A, Monteiro A. Doublesex mediates the development of sex-specific pheromone organs in Bicyclus butterflies via multiple mechanisms. Mol Biol Evol. 2020:msaa039.

27. Friedländer M. Control of the eupyrene-apyrene sperm dimorphism in Lepidoptera. J Insect Physiol. 1997;43:1085-92.

28. Friedländer M, Seth RK, Reynolds SE. Eupyrene and apyrene sperm: dichotomous spermatogenesis in Lepidoptera. Adv Insect Physiol. 2005;32: 206-308.

29. Chen $K$, Chen S, Xu J, Yu Y, Liu Z, Tan A, Huang Y. Maelstrom regulates spermatogenesis of the silkworm, Bombyx mori. Insect Biochem Mol Biol. 2019;109:43-51.

30. Zhang P, Zhong J, Cao G, Xue R, Gong C. BmAly is an important factor in meiotic progression and spermatid differentiation in Bombyx mori (Lepidoptera: Bombycidae). J Insect Sci. 2014;14:188.

31. Suzuki MG, Ohbayashi F, Mita K, Shimada T. The mechanism of sex-specific splicing at the doublesex gene is different between Drosophila melanogaster and Bombyx mori. Insect Biochem Mol Biol. 2001;31:1201-11.

32. Singh NK, Singh NN, Androphy EJ, Singh RN. Splicing of a critical exon of human survival motor neuron is regulated by a unique silencer element located in the last intron. Mol Cell Biol. 2006;26:1333-46.

33. Lorson $\mathrm{CL}$, Rindt $\mathrm{H}$, Shababi M. Spinal muscular atrophy: mechanisms and therapeutic strategies. Hum Mol Genet. 2010;19:R111-8.

34. Bebee TW, Gladman JT, Chandler DS. Splicing regulation of the survival motor neuron genes and implications for treatment of spinal muscular atrophy. Front Biosci. 2010;15:1191-204.

35. Waterbury JA, Jackson LL, Schedl P. Analysis of the doublesex female protein in Drosophila melanogaster: role on sexual differentiation and behavior and dependence on intersex. Genetics. 1999;152:1653-67.

36. Soper SFC, Heijden GWVD, Hardiman TC, Goodheart M, Martin SL, Boer PD, et al. Mouse maelstrom, a component of nuage, is essential for spermatogenesis and transposon repression in meiosis. Dev Cell. 2008;15: 285-97

37. Pek JW, Lim AK, Kai T. Drosophila maelstrom ensures proper germline stem cell lineage differentiation by repressing microrna-7. Dev Cell. 2009;17:417-24.

38. White-Cooper H, Schafer MA, Alphey LS, Fuller MT. Transcriptional and posttranscriptional control mechanisms coordinate the onset of spermatid differentiation with meiosis I in Drosophila. Development. 1998:125:125-34.

39. White-Cooper H, Leroy D, MacQueen A, Fuller MT. Transcription of meiotic cell cycle and terminal differentiation genes depends on a conserved chromatin associated protein, whose nuclear localisation is regulated. Development. 2000;127:5463-73.

40. Arunkumar KP, Mita K, Nagaraju J. The silkworm Z chromosome is enriched in testis-specific genes. Genetics. 2009;182:493-501.

41. Zha X, Xia Q, Duan J, Wang C, He N, Xiang Z. Dosage analysis of Z chromosome genes using microarray in silkworm, Bombyx mori. Insect Biochem Mol Biol. 2009:39:315-21.

42. Katsuma S, Sugano Y, Shimada T. Two conserved cysteine residues are required for the masculinizing activity of the silkworm Masc protein. J Bio Chem. 2015;290:26114-24

43. Qin Z, Juan L, Mao W, He W, Yao W, Kai W, et al. A novel splice variant of the masulinizing gene Masc with piRNA-cleavage-site defect functions in female external genital development in the silkworm, Bombyx mori. Biomolecules. 2019;9:318

44. Sumitani M, Sakurai T, Kasashima K, Kobayashi S, Uchino K, Kanzaki R, et al. Establishment of a specific cell death induction system in Bombyx mori by a transgene with the conserved apoptotic regulator, mouse Bcl-2-associated $X$ protein (mouse Bax). Insect Mol Biol. 2015;24:671-80.

45. Takasu Y, Sajwan S, Daimon T, Osanai-Futahashi M, Uchino K, Sezutsu H, et al. Efficient TALEN construction for Bombyx mori gene targeting. PLoS One. 2013;8:e73458

46. Kasahara R, Fugaku A, Suzuki MG. Deficiency in dmrt99B ortholog causes behavioral abnormalities in the silkworm, Bombyx mori. Appl Entomol Zool. 2018:53:381-93. 
47. Daimon T, Kiuchi T, Takasu Y. Recent progress in genome engineering techniques in the silkworm, Bombyx mori. Develop Growth Differ. 2014;56:14-25.

48. Suzuki MG, Suzuki K, Aoki F, Ajimura M. Effect of RNAi-mediated knockdown of the Bombyx mori transformer-2 gene on the sex-specific splicing of Bmdsx pre-mRNA. Int J Dev Biol. 2012;56:693-9.

49. Koike Y, Mita K, Suzuki MG, Maeda S, Abe H, Osoegawa K, et al. Genomic sequence of a 320-kb segment of the Z chromosome of Bombyx mori containing a kettin ortholog. Mol Gen Genomics. 2003;269:137-49.

50. Aslam AF, Kiya T, Mita K, Iwami M. Identification of novel bombyxin genes from the genome of the silkmoth Bombyx mori and analysis of their expression. Zool Sci. 2011:28:609-16.

51. Kim YJ, Zitnan D, Cho KH, Schooley DA, Mizoguchi A, Adams ME. Central peptidergic ensembles associated with organization of an innate behavior. Proc Natl Acad Sci U S A. 2006;103:14211-6.

52. Takase D, Suzuki MG. Analysis of sex-specific regulation of the dunce gene in the Drosophila melanogaster central nervous system. Cytologia. 2018;83: $345-6$.

53. Tanaka A, Aoki F, Suzuki MG. Conserved domains in the transformer protein act complementary to regulate sex-specific splicing of its own pre-mRNA. Sex Dev. 2018;12:180-90

\section{Publisher's Note}

Springer Nature remains neutral with regard to jurisdictional claims in published maps and institutional affiliations.

Ready to submit your research? Choose BMC and benefit from:

- fast, convenient online submission

- thorough peer review by experienced researchers in your field

- rapid publication on acceptance

- support for research data, including large and complex data types

- gold Open Access which fosters wider collaboration and increased citations

- maximum visibility for your research: over $100 \mathrm{M}$ website views per year

At BMC, research is always in progress.

Learn more biomedcentral.com/submissions 